Trends In Ecology \& Evolution

August 2019, Volume 34 Issue 8 Pages 688-689

https://doi.org/10.1016/j.tree.2019.05.011

https://archimer.ifremer.fr/doc/00503/61431/

\title{
Long-Distance Marine Connectivity: Poorly Understood but Potentially Important
}

\author{
Manel Stéphanie ${ }^{1,{ }^{*}}$, Loiseau Nicolas ${ }^{2,3}$, Puebla Oscar ${ }^{4}$
}

1 PSL Research University, EPHE, CNRS, UM, UM3, IRD, UMR 5175 CEFE, Montpellier, France

2 MARBEC, Univ Montpellier, CNRS, Ifremer, IRD, Montpellier, France

3 University Grenoble Alpes, CNRS, Univ. Savoie Mont Blanc, LECA, Laboratoire 9 d'Ecologie Alpine, F-38000 Grenoble, France

${ }^{4}$ GEOMAR Helmholtz Centre for Ocean Research Kiel, Marine Evolutionary Ecology, Düsternbrooker Weg 20, 24105, Kiel, Germany

* Corresponding author email address : stephanie.manel@cefe.cnrs.fr

Keywords : connectivity ; Dispersal ; marine protected areas ; spatial planning 
21 We agree with Costello \& Connor [1] that most marine reserves should not be considered as totally isolated islands. On the other hand, the marine environment is not a homogeneous habitat either. Landscape connectivity quantifies the extent to which the movements of genes, propagules and individuals between populations (i.e. dispersal) are facilitated or hindered by the structure and composition of the landscape [2]. Landscape [3] and seascape [4,5] genetics in particular provide a framework (concepts, tools, community) that considers explicitly the suitability of habitats outside marine reserves for the estimation of genetic connectivity [6]. Yet, although tools are available (Box 1), the vast majority of studies that estimate connectivity in the context of marine conservation and strategic management planning still ignore seascape resistance models (Box 1). In this respect, it is true that marine reserves in particular, and marine populations more generally are often envisioned as islands. We advocate for sampling designs that are more continuous across the landscape, including areas and populations inside and outside marines reserves but also in between (e.g. [7]).

\section{Long-distance connectivity is poorly understood}

Since the contribution of long distances (i.e. $>40 \mathrm{~km}$ ) to marine dispersal kernels is currently unknown [8], we cannot yet provide strong recommendations for the spacing among marine reserves on the basis of long-distance connectivity. We suggest that long-distance dispersal is likely to have been underestimated [8]. However, this does not necessarily imply that it is important from a demographic perspective, nor that the role of local retention and local dispersal, for which evidence has accumulated over the last two decades, is not. Furthermore, long-distance dispersal events are expected to be rare, which has implications at different spatial and temporal scales than local dispersal events. On the other hand, long-distance 
connectivity could also result from more frequent stepping-stone dispersal events over several generations. By no means, does our review suggests that dispersal, because it is spatially extensive, is not important for conservation and management planning. We believe, on the contrary, that seascape connectivity studies are needed to advise on the minimal distance among marine reserves. In this respect, we concur with Costello \& Connor [1] that future connectivity studies should account for the seabed and water column habitat, as well as fishing pressure within these habitats. Seascape genetics opens promising perspectives to do so. It will also open perspectives to forecast the effect of climate change on species range shifts accounting for connectivity [9].

\section{Long-distance connectivity is potentially important for threatened marine biodiversity}

Many marine species are overexploited and threatened [10]. Long-distance dispersal can potentially connect distant and isolated populations within marine reserves and sustain biodiversity and biomass in exploited areas located more than $40 \mathrm{~km}$ from marine reserve boundaries. Rare long-distance dispersal from marine reserves due to a few successful migrants can also be sufficient to re-colonize areas where local populations have been extirpated, or expand species distributions in response to global change. Long-distance dispersal between populations that are genetically differentiated or locally adapted can moreover contribute to limiting inbreeding, increasing genetic diversity, and facilitating adaptation to a changing environment. One open question here is to what extent long-distance dispersal contributes to connect the largest marine reserves $\left(>1000 \mathrm{~km}^{2}\right)$ in the global network, which can provide the greatest benefits but are also the most isolated ones (closest reserves $>359 \mathrm{~km})[8]$. 


\section{Spatial planning should be multi-specific and multi-objectives}

68 We agree with Costello \& Connor [1] that planning should focus on area and creation of

69 reserve networks that contain representative range of habitat. However, without consideration

70 for connectivity, efficiency of marine protected networks will be lower. A recent conservation

71 planning study showed that combining representation and connectivity objectives provides the

72 best strategy for enhanced biodiversity persistence in network of marine reserves [11].

73 Therefore, we do not recommend disregarding connectivity in conservation and management

74 planning, but instead to develop multi-species and multi-objective strategies. Such

75 applications are still scarce not only in marine but also terrestrial systems, but again the tools

76 are available [11]. We agree with Costello \& Connor [1] that such multi-objective strategies

77 should also account for a representative range of benthic and pelagic habitats within

78 biogeographic realms [12].

79 
Seascape genetics integrates the concepts and tools used to describe the patterns and understand the processes of marine connectivity in a spatially explicitly context that considers habitat characteristics.

(i) The first step to estimate marine connectivity in a seascape genetics context is the assessment of resistance surfaces from seascape variables. Resistance surfaces are spatial layers that represent the extent to which the conditions in each grid cell covering the study area constrain movement or gene flow ([6], chap 8, p129-144). Seascape variables include current flow, habitat, climatic variables, bathymetry, chlorophyll etc, as well as fishing data that are increasingly publicly available ${ }^{1}$. Seascape variables are then converted to resistance cost surfaces and costs are assigned ranging from 1 (no resistance to movement) to 100 (strong barriers to movement). Cost can be determined by expert opinion or estimated from genetic data.

(ii) A second step is the conversion of the resistance surface into a seascape connectivity measure (i.e. a matrix of connectivity distances). Such conversion is typically based on leastcost paths or circuit theory ([6], chap 8, p129-144). Least-cost analysis identifies the least costly route that an organism can take from one area to another, while the circuit theory approach ([6], chap 8, p129-144) calculates resistance matrices between populations or individuals and estimates all potential movement pathways across the landscape based on the cumulative cost of movement due to landscape resistance.

(iii) Finally, the connectivity measure resulting from resistance surface is correlated to genetic distances to detect statistically significant seascape drivers of genetic differentiation and to select the most important drivers to be used for estimating resistance surfaces. Various 
approaches can be used to assess the correlation between genetic distance and multiple

107

108

109

110

111

112

113

114

115

116

117

118

119

120

$121571-573$

122 3. Manel, S. et al. (2003) Landscape genetics: combining landscape ecology and population

123 genetics. Trends in Ecology and Evolution 18, 157-206.

124

125 marine connectivity. Mar Ecol Prog Ser 554

126 5. Riginos, C. et al. (2016) Navigating the currents of seascape genomics: how spatial

127 analyses can augment population genomic studies. Current Zoology 62, 581-601.

128 6. Balkenhol, N. et al. (2016) Landscape genetics, John Wiley \& Sons Ltd.

129 7. Puebla, O. et al. (2012) On the spatial scale of dispersal in coral reef fishes. Molecular

130 Ecology 21, 5675-5688. 
131 8. Manel, S. et al. (2019) Long-Distance Benefits of Marine Reserves: Myth or Reality?

132 Trends in Ecology \& Evolution 34, 342-354.

133 9. Razgour, O. et al. (2019) Considering adaptive genetic variation in climate change

134 vulnerability assessment reduces species range loss projections. Proceedings of the National

135 Academy of Sciences, 201820663.

136 10. O'Hara, C.C. et al. (2019) Mapping status and conservation of global at-risk marine

137 biodiversity. Conservation Letters 0, e12651.

138 11. Magris, R.A. et al. (2018) Biologically representative and well-connected marine reserves

139 enhance biodiversity persistence in conservation planning. Conservation Letters 11.

140 12. Costello, M.J. et al. (2017) Marine biogeographic realms and species endemicity. Nature

141 Communications 8, 1057. 